\title{
Association of depressive symptoms and risk of knee pain: the moderating effect of sex
}

\author{
Haiyan $\mathrm{Hu}^{1 \dagger}$, Wenjun Liu ${ }^{2,3+}$, Yang Liu ${ }^{2,3}$, Jay Pan ${ }^{1,4^{*}}$ and Xiaozuo Zheng ${ }^{5^{*}}$
}

\begin{abstract}
Background: Depression has been shown in some studies to be associated with knee pain. Females were widely recognized as more vulnerable to depression and knee pain than males. However, the role of sex in this correlation was under-researched. This study aimed to investigate the association between depressive symptoms and subsequent knee pain, as well as whether and how sex would moderate this association based on a four-wave (Wave 1 in 2010-2011, Wave 2 in 2013, Wave 3 in 2015, and Wave 4 in 2018) longitudinal study among middle-aged and elderly Chinese adults.

Methods: Seventeen thousand seven hundred eight participants were recruited and followed in the China Health and Retirement Longitudinal Study (CHARLS). Ten thousand four hundred fifty-one entered the final analysis based on the inclusion and exclusion criteria. Knee pain was assessed by self-report. Depressive symptoms were evaluated using the validated 10-item Center for Epidemiological Studies-Depression Scale (CESD-10). Cox proportional hazards models were used to calculate hazard ratios with 95\% confidence intervals (Cls) after controlling potential confounders to examine the association between depressive symptoms and subsequent incident and persistent knee pain. Non-linear association of depressive symptoms score (CESD-10) and risk of knee pain was also investigated via applying 3-knotted restricted cubic spline regression. An interaction term of depressive symptoms status and sex was added to investigate the moderating effect of sex on the relationship between depressive symptoms status and the risk of knee pain.

Results: The median follow-up time was seven years for all the outcomes. Participants with depressive symptoms were 1.45 times (95\% Cl: 1.34-1.56) and 2.16 times (95\% Cl: 1.85-2.52) more likely to develop the incident and persistent knee pain after multivariable were adjusted, compared with those without depressive symptoms. There was a non-linear association between CESD-10 score and risk of knee pain. Compared with females, males had an enhanced correlation between depressive symptoms status and knee pain (multivariable-adjusted HR: 1.22, 95\% Cl: 1.05-1.42 and HR: $1.57,95 \% \mathrm{Cl}: 1.14-2.17$ for the incident and persistent knee pain, respectively).
\end{abstract}

Conclusion: Depressive symptoms are independently associated with an excess risk of knee pain, with a stronger correlation for males than females among middle-aged and elderly Chinese adults.

\footnotetext{
*Correspondence: panjie.jay@scu.edu.cn; xiaozuo_zheng@163.com

${ }^{\dagger} \mathrm{H}$ aiyan $\mathrm{Hu}$ and Wenjun Liu contributed equally to this article and should

be considered co-first authors.

${ }^{1}$ HEOA Group, West China School of Public Health and West China

Fourth Hospital, Sichuan University, No. 16, Section 3, Ren Min Nan Road,

Chengdu 610041, China

${ }^{5}$ Department of Orthopaedic Surgery, Third Hospital of Hebei Medical

University, No.139 Ziqiang Road, Shijiazhuang 050051, Hebei, China

Full list of author information is available at the end of the article
}

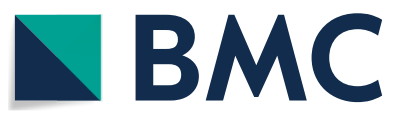

(C) The Author(s) 2021. Open Access This article is licensed under a Creative Commons Attribution 4.0 International License, which permits use, sharing, adaptation, distribution and reproduction in any medium or format, as long as you give appropriate credit to the original author(s) and the source, provide a link to the Creative Commons licence, and indicate if changes were made. The images or other third party material in this article are included in the article's Creative Commons licence, unless indicated otherwise in a credit line to the material. If material is not included in the article's Creative Commons licence and your intended use is not permitted by statutory regulation or exceeds the permitted use, you will need to obtain permission directly from the copyright holder. To view a copy of this licence, visit http://creativecommons.org/licenses/by/4.0/. The Creative Commons Public Domain Dedication waiver (http://creativeco mmons.org/publicdomain/zero/1.0/) applies to the data made available in this article, unless otherwise stated in a credit line to the data. 


\section{Plain English Summary}

This study investigated the incidence of knee pain, the correlation of depressive symptoms with knee pain, and the moderating effect of sex on this correlation in a nationally representative cohort of middle-aged and elderly Chinese adults. Results suggested that depressive symptoms were associated with an excess risk of knee pain, with a stronger correlation for males than females.

Keywords: Knee pain, Musculoskeletal pain, Chronic pain, Depressive symptoms, Mental disorder, Cohort study, Moderation analysis, Moderating effect, Sex

\section{Highlights}

Depressive symptoms significantly increase the risk of knee pain.

Females are more vulnerable to depression and knee pain than males.

The correlation between depressive symptoms and knee pain is more significant in males than in females.

\section{Introduction}

Knee pain, one of the most prevalent musculoskeletal (MSK) pain disorders, affects large proportions of the population [1]. It was estimated that approximately $33 \%$ of middle-aged and elderly adults were affected by painful knees [2, 3]. Its prevalence has increased by almost $65 \%$ over the past two decades [1] and is expected to continue as life expectancies grow. Chronic knee pain is commonly defined as knee pain that persists longer than three months or beyond the normal tissue healing duration [4]. It is a leading cause of disability, one of the main reasons for medical treatment-seeking behavior in primary care settings, and contributes to a substantial socioeconomic burden [5]. However, the incidence of knee pain among the general population remains underresearched. Identifying the significant risk factors for knee pain is valuable for establishing prevention plans for the people at risk and providing clinical evidence to develop more effective management strategies for knee pain sufferers from progressing into chronic complaints.

In the most widely referenced biopsychosocial framework, both intra-articular and extra-articular risk factors may lead to knee pain [5-7]. Psychological factors are frequently recognized as correlated with knee pain [5, $8]$, with depression being recognized as one of the most common and disease-laden psychological disorders, particularly for the middle-aged and elderly population. For the 50-74 age group, the percentage change in the number of disability-adjusted life-years (DALYs) attributing to depression has risen by $107.3 \%$ over the past three decades [9]. Previous studies have suggested that an increase in inflammatory cytokines and altered neurotransmitter levels associated with depression contributes to changing physical pain perception threshold, while the underline mechanism was not fully understood [10-12]. Therefore, depression may aggravate the perception of knee pain.

Depression has been shown in some studies to be associated with knee pain [13-22]. Some detected a significant association [16-20,22], while others failed to [13-15, 21]. Inherent heterogeneity including, but not limited to, study design, sample size, sample selection, and statistical modeling method may have contributed to this variation in the observed depression-knee pain correlation. Besides, whether and how sex moderated the relationship between depression and knee pain was not well-characterized. Although females have been widely perceived as more vulnerable to depression [23, 24] and pain disorders $[25,26]$, sex is often examined as an averaged effect in previous studies [16, 17, 19, 20]. More specific investigation into the role of sex in the depression-knee pain relationship would improve knee pain prevention, care, and management for both sexes.

This study aimed to 1) investigate the incidence rate of knee pain among middle-aged and elderly adults according to the status of the depressive symptoms, 2) examine the correlation of depressive symptoms and subsequent knee pain, and 3) explore the moderating role of sex on this relationship.

\section{Methods Study design}

Data used in this study were obtained from the China Health and Retirement Longitudinal Study (CHARLS), available to the public at http://charls.pku.edu.cn/index/ en.html after registration and application. CHARLS was an ongoing nationally representative populationbased cohort survey in China, harmonized with the US Health and Retirement Study (HRS) and related surveys on aging worldwide [27]. Zhao et al'. described details for the design and performance of CHARLS [28]. The Biomedical Ethics Review Committee of Peking University approved the CHARLS study (No: IRB0000105211,015) [28]. Ethics approval for the use of CHARLS data 
was obtained from the University of Newcastle Human Research Ethics Committee (H-2015-0290).

\section{Participants and data collection}

Seventeen thousand seven hundred eight participants from 28 provinces, municipal cities, and autonomous regions were recruited through the multistage probability-proportional-to-size (PPS) sampling strategy in the baseline survey (Wave 1, between June 2011 and March 2012). Follow-ups were performed in Wave 2 (2013), Wave 3 (2015), and Wave 4 (2018) [27]. Participants reflect the middle-aged and elderly Chinese population collectively [27]. Respondents were interviewed by trained staff using face-to-face laptop-assisted questionnaires [28]. The response rate for the baseline survey (Wave 1) was $80.5 \%$. CHARLS interviewers obtained informed consent from all participants before the survey [27].

\section{Measures \\ Knee pain}

The study outcome was an incident of knee pain. In the questionnaires, knee pain was assessed by asking whether the participants had experienced knee pain in the past month. Following previous studies [29, 30], participants were classified into two subgroups: persistent and incident knee pain. Participants with persistent knee pain refer to those reporting knee pain during two or more consecutive follow-up waves. Participants with incident knee pain refer to those subjects with pain in one or more non-consecutive waves.

\section{Depressive symptoms}

Depressive symptoms were evaluated using the validated Center for Epidemiological Studies-Depression Scale (CESD-10), which rates symptoms related to depression $[31,32]$. Ten items' summation score may range from 0 to 30 , with higher values indicating more severe depressive symptoms. A total CESD-10 score of 10 or greater was commonly used to indicate clinically significant depression for Chinese adults [29].

\section{Covariates}

Consistent with previous studies [17, 29, 33], the following covariables were included. The interviewer-administered questionnaire obtained all the covariates at the baseline survey.

a) Demographic characteristics included age (continuous: years) and sex (binary: male or female). In the moderation analysis, sex was considered as the moderator. b) Socioeconomic status (SES) included education attainment (ordinal: illiterate, primary school, middle school, high school or above), marital status (binary: currently married or partnered, or single), neighborhood (binary: rural or urban), household income per capita (ordinal: categorized into four quartiles [Qs], from Q1[the lowest] to Q4 [the highest]), occupation (binary: non-farming or farming).

c) Health status included the number of common chronic comorbidities, body mass index (BMI) level, and injury history (binary: with or without). The number of common chronic comorbidities (ordinal: none, one, two, three, or above). Chronic diseases involved hypertension, diabetes, dyslipidemia, chronic lung diseases, liver diseases, heart disease, stroke, cancer, chronic kidney diseases, digestive diseases, etc. Following the Working Group on Obesity in China, body mass indexes (BMI) were categorized into four levels (ordinal: underweight $[\mathrm{BMI}<18.5 \mathrm{~kg} /$ $\left.\mathrm{m}^{2}\right]$, normal $\left[18.5 \leq \mathrm{BMI}<24 \mathrm{~kg} / \mathrm{m}^{2}\right]$, overweight $\left[24 \leq \mathrm{BMI}<28 \mathrm{~kg} / \mathrm{m}^{2}\right]$, and obesity $[\mathrm{BMI} \geq 28 \mathrm{~kg} /$ $\mathrm{m}^{2}$ ] [34]. Injury history was assessed by asking the respondents "Have you ever been in a traffic accident or any other kind of major accidental injury and received medical treatment?". If the respondent said yes to the question, the respondent was classified as having injury history.

d) Health-related behavior included cigarette smoking (binary: yes or no) and drinking (ordinal: none, less than once a month or more than once a month).

\section{Research samples}

For this analysis, we selected participants who were followed up for at least one wave since the baseline. We excluded participants without baseline information for socio-demographics, health and health-related behavior, knee pain or depressive symptoms, and those reporting baseline knee pain. Figure 1 presents the flow chart of screening the research population for this study.

\section{Statistical analysis}

Data were described as means with standard deviations (SDs) for quantitative variables and frequency with proportions for binary and ordinal variables. Baseline characteristics were summarized according to the presence of depressive symptoms. Between-group comparisons were conducted according to depressive symptoms using the Student's T-test, Chi-square test, and the Wilcoxon ranksum test, as appropriate.

The person-year of follow-up for each respondent was calculated from the baseline wave (2011-2012) to the years of confirming knee pain, fail to follow-up, or the 


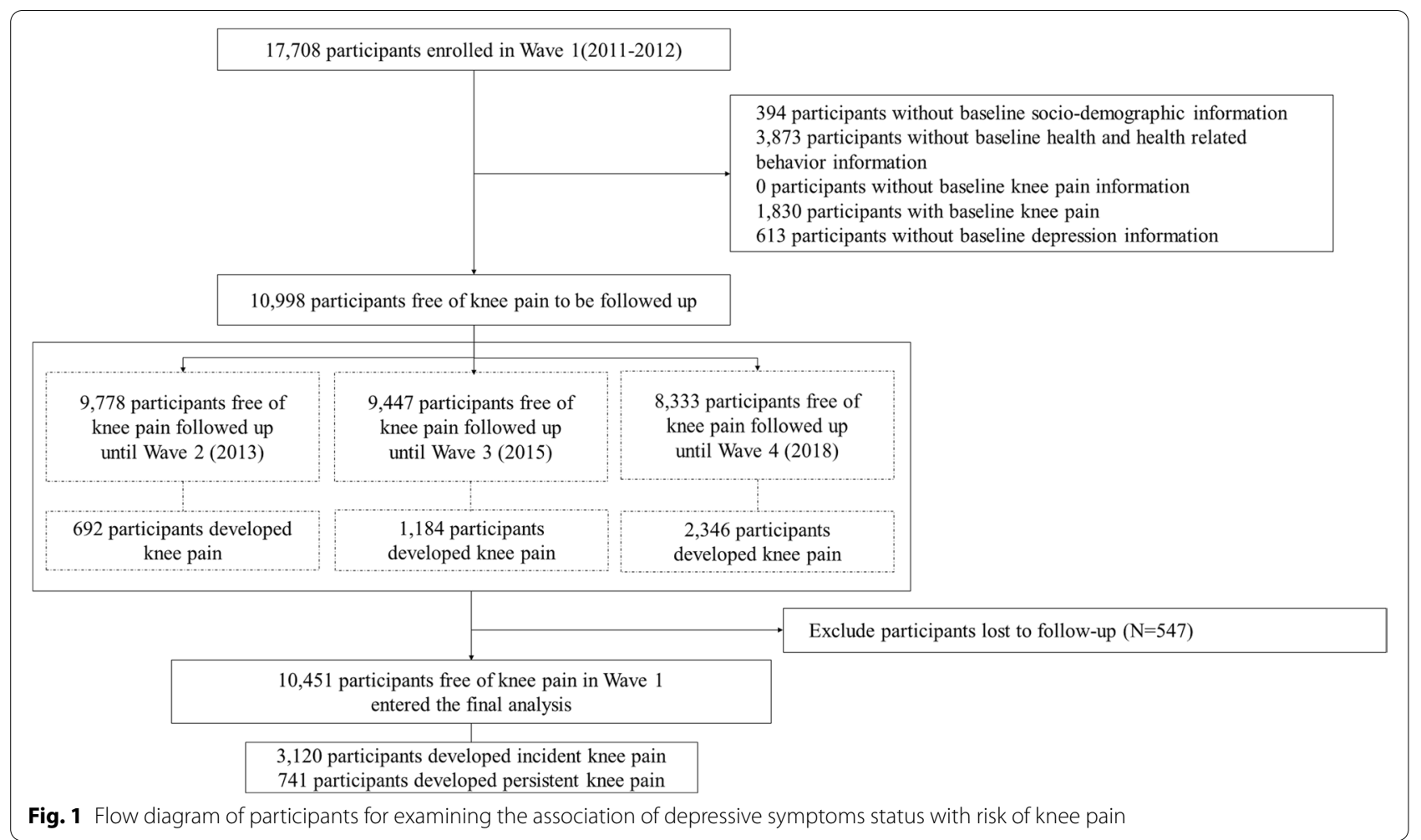

end of follow-up, whichever came first. The incidence rates per 1,000 person-years and 95\% confidence intervals (CIs) of the incident and persistent knee pain were calculated as a whole and by baseline depression status.

To examine the association between depressive symptoms and subsequent knee pain, Cox proportional hazards models were used to calculate hazard ratios (HRs) with 95\% CIs. We reported both the unadjusted model (crude model) and the covariables-adjusted model (adjusted model) in the main text. Schoenfeld residuals were tested for evaluating the proportional hazards assumption for Cox proportional hazards regression, using the "estat phtest" command in Stata software. A $p$-value larger than 0.05 indicates that there is no evidence that the proportional-hazards assumption has been violated. Only regression models satisfying this assumption were reported in the text. Besides, we explored the potential non-linear associations of depressive symptoms score (CES-D) with the incident and persistent knee pain in the adjusted model applying 3-knotted restricted cubic spline regression through the Stata command "mkspline". A $p$-value of less than 0.05 for test linearity hypotheses after estimation indicates that the null hypothesis would be rejected, and there would be a non-linear association.

$26.56 \%(4,368$ of 16,447$)$ of total observations containing missing values were discarded from the initial analysis, known as listwise deletion or complete case analysis.
To account for this method's significant drawback, this study conducted multiple imputations of chained equations approach based on the baseline characteristics, followed by Cox proportional hazards regression modeling. Under the assumption that these missing values are missing at random (MAR), a simulation-based threestep imputation procedure was conducted. First, missing values were replaced with multiple sets of imputed values from the regress, logit, or ordinal logit model as appropriate to form fifty completed datasets. Second, standard complete data analyses were performed based on each dataset imputed from step 1 . Third, results from step 2 were consolidated into multiple imputation inferences using the Stata command "mi estimate" [35].

All statistical analyses were performed in Stata 16 (StataCorp LP, College Station, Texas), and the significance was set at a two-tailed $\alpha$ of less than 0.05 .

\section{Results}

\section{Participants' characteristics}

Table 1 presents the participants' baseline characteristics. A total of 10,451 participants entered the final analysis, of which 3,368 (32.23\%) were depressed at baseline. The mean age was 59.45 years (SD: 9.58 years). Participants were mostly female $(N=5,349,51.18 \%)$, educated less than six years (illiterate: $N=2,653$, 25.39\%; primary school: $N=4,239,40.56 \%$ ), currently 
Table 1 Baseline characteristic of study participants according to depressive symptoms status $(N=10,451)$

\begin{tabular}{|c|c|c|c|c|}
\hline & \multicolumn{2}{|c|}{ Depressive symptoms } & \multirow[t]{2}{*}{ Total $(N=10,451)$} & \multirow[t]{2}{*}{$p$ value } \\
\hline & With $(N=3,368)$ & Without $(N=7,083)$ & & \\
\hline Age, mean (SD), years & $60.19(9.72)$ & $59.10(9.49)$ & $59.45(9.58)$ & $<0.001$ \\
\hline Sex, $n(\%)$ & & & & $<0.001$ \\
\hline Female & $2,020(59.98)$ & $3,329(47.00)$ & $5,349(51.18)$ & \\
\hline Male & $1,348(40.02)$ & $3,754(53.00)$ & $5,102(48.82)$ & \\
\hline Education attainment, $\mathrm{n}(\%)$ & & & & $<0.001$ \\
\hline Illiterate & $1,075(31.92)$ & $1,578(22.28)$ & $2,653(25.39)$ & \\
\hline Primary school & $1,483(44.03)$ & $2,756(38.91)$ & $4,239(40.56)$ & \\
\hline Middle school & $600(17.81)$ & $1,708(24.11)$ & $2,308(22.08)$ & \\
\hline High school or above & $210(6.24)$ & $1,041(14.70)$ & $1,251(11.97)$ & \\
\hline Marital status, n (\%) & & & & $<0.001$ \\
\hline Otherwise (divorced, widowed, never married) & $512(15.20)$ & $645(9.11)$ & $1,157(11.07)$ & \\
\hline Currently married or partnered & $2,856(84.80)$ & $6,438(90.89)$ & $9,294(88.93)$ & \\
\hline Type of neighborhood, n (\%) & & & & $<0.001$ \\
\hline Rural & $2,876(85.39)$ & $5,485(77.44)$ & $8,361(80.00)$ & \\
\hline Urban & $492(14.61)$ & $1,598(22.56)$ & $2,090(20.00)$ & \\
\hline Household income per capita, n (\%) & & & & $<0.001$ \\
\hline Quantile 1 (lowest) & $1,088(32.30)$ & $1,524(21.52)$ & $2,612(24.99)$ & \\
\hline Quantile 2 & $945(28.06)$ & $1,668(23.55)$ & $2,613(25.00)$ & \\
\hline Quantile 3 & $770(22.86)$ & $1,843(26.02)$ & $2,613(25.00)$ & \\
\hline Quantile 4 (highest) & $565(16.78)$ & $2,048(28.91)$ & $2,613(25.00)$ & \\
\hline Occupation, n (\%) & & & & $<0.001$ \\
\hline Non-farming & $1,310(38.90)$ & $3,024(42.69)$ & $4,334(41.47)$ & \\
\hline Farming & $2,058(61.10)$ & $4,059(57.31)$ & $6,117(58.53)$ & \\
\hline Body mass index (BMI) level, n (\%) & & & & $<0.001$ \\
\hline Underweight $(\mathrm{BMI}<18.5)$ & $286(8.49)$ & $354(5.00)$ & $640(6.12)$ & \\
\hline Normal $(18.5 \leq \mathrm{BMI}<24)$ & $1,885(55.97)$ & $3,666(51.76)$ & $5,551(53.11)$ & \\
\hline Overweight $(24 \leq \mathrm{BMI}<28)$ & $888(26.37)$ & $2,189(30.90)$ & $3,077(29.44)$ & \\
\hline Obesity (BMI $\geq 28)$ & $309(9.17)$ & $874(12.34)$ & $1,183(11.32)$ & \\
\hline Cigarette smoking, n (\%) & & & & $<0.001$ \\
\hline No & $2,417(71.76)$ & $4,757(67.16)$ & $7,174(68.64)$ & \\
\hline Yes & $951(28.24)$ & $2,326(32.84)$ & $3,277(31.36)$ & \\
\hline Drinking, n (\%) & & & & $<0.001$ \\
\hline None & $2,439(72.42)$ & $4,511(63.69)$ & $6,950(66.50)$ & \\
\hline Less than once a month & $232(6.89)$ & $599(8.46)$ & $831(7.95)$ & \\
\hline More than once a month & $697(20.69)$ & $1,973(27.86)$ & $2,670(25.55)$ & \\
\hline Injury, n (\%) & & & & $<0.001$ \\
\hline Without & $3,163(93.91)$ & $6,859(96.84)$ & $10,022(95.90)$ & \\
\hline With & 205(6.09) & 224(3.16) & $429(4.10)$ & \\
\hline Number of chronic diseases, n (\%) & & & & $<0.001$ \\
\hline None & $878(26.07)$ & $2,879(40.65)$ & $3,757(35.95)$ & \\
\hline One & $1,046(31.06)$ & $2,254(31.82)$ & $3,300(31.58)$ & \\
\hline Two & $727(21.59)$ & $1,181(16.67)$ & $1,908(18.26)$ & \\
\hline Three or above & $717(21.29)$ & $769(10.86)$ & $1,486(14.22)$ & \\
\hline
\end{tabular}

Notes: SD standard deviation 
married or partnered $(N=9,294,88.93 \%)$, living in rural areas $(N=8,361,80.00 \%)$, normal weighted $(N=5,551$, $53.11 \%)$, non-smokers $(N=7,174,68.64 \%)$, non-drinkers $(N=6,950,66.50 \%)$, without injury history $(N=10,022$, 95.90\%) and suffered from one or more chronic diseases $(N=6,694,64.05 \%)$. Group comparison results showed that depressive symptoms were more prevalent in the senior, female, less educated, divorced, widowed or never married, non-smokers, non-drinkers, and those living in rural areas, economically disadvantaged, engaged in agriculture, with injury history, and suffering from chronic diseases $(p<0.001)$.

\section{Incidence rate and hazard ratio of knee pain according to depressive symptoms status}

Ten thousand four hundred fifty-one respondents were followed up (median: 7 years). A total of 3,120 developed incident knee pain, with the IR being 46.44, 65.13, and 37.66 per 1000 person-years for all the respondents, respondents with and without depressive symptoms, respectively (Table 2, panel A). A total of 741 developed persistent knee pain, with the IR being 14.5, 28.51, and 8.69 per 1000 person-years for all the respondents, respondents with and without depressive symptoms, respectively (Table 2 , panel $\mathrm{B}$ ).

Participants with depressive symptoms were consistently at higher risk of developing incident knee pain (crude HR: 1.75, 95\% CI [1.63-1.88]; adjusted HR: 1.45, 95\% CI [1.34-1.56], Table 2 panel A) and persistent knee pain (crude HR: 3.33, 95\% CI [2.88-3.85]; adjusted HR: 2.16, 95\% CI [1.85-2.52], Table 2 panel B). Multiple imputations inference of depression's contribution did not show appreciable changes for incident knee pain (adjusted HR: 1.39, 95\% CI [1.32-1.48]) or persistent knee pain (adjusted HR:2.09, 95\% CI [1.86-2.34]).

\section{Non-linear association of depressive symptoms scores} with knee pain

A non-linear and positive association between the CES-D total score and risk of knee pain using restricted cubic spline regression was found (for linearity, Chi-square of Wald tests $=26.30, p<0.001$ for incident knee pain; Chisquare of Wald tests $=14.31, p<0.001$ for persistent knee pain (Fig. 2).

\section{Moderating effect of sex on the relationship between depressive symptoms status and knee pain} Significant HRs greater than 1.00 were observed for interactions of depressive symptoms and sex. These results indicated that the correlation of depressive symptoms with subsequent incident knee and persistent knee pain was 1.22 (95\% CI: 1.05-1.42) and 1.57 (95\% CI: $1.14-$ 2.17) times stronger for males compared with females, respectively (Table 3 , panel A and $\mathrm{B}$ ). Results of moderating effect analyses were robust when multiple imputations were performed for incident knee pain (adjusted HR: $1.21,95 \%$ CI [1.08-1.36]) and persistent knee pain (adjusted HR: 1.48, 95\% CI [1.16-1.89]).

\section{Discussion}

The main findings were three-fold. First, incidence rates of knee pain in participants with depressive symptoms were consistently higher than those without depressive symptoms. Second, depressive symptoms were independently associated with the development of knee pain after controlling potential confounders. Third, sex moderated this association where depressed males were at a higher risk of developing future knee pain than depressed females.

Table 2 Incidence rate and hazard ratio of knee pain according to depressive symptoms status

\begin{tabular}{|c|c|c|c|c|}
\hline \multirow[t]{2}{*}{ Outcome } & \multirow[t]{2}{*}{ Case / person-years } & \multirow{2}{*}{$\begin{array}{l}\text { Incidence rate, per } 1000 \\
\text { person-years }\end{array}$} & \multicolumn{2}{|l|}{$\mathrm{HR}(95 \% \mathrm{Cl})$} \\
\hline & & & Crude model & Adjusted model \\
\hline \multicolumn{5}{|c|}{ Panel A: Incident knee pain } \\
\hline \multicolumn{5}{|c|}{ Depressive symptoms } \\
\hline Without & $1721 / 45701$ & 37.66 & 1 [Reference] & 1 [Reference] \\
\hline With & $1399 / 21480$ & 65.13 & $1.75(1.63-1.88)$ & $1.45(1.34-1.56)$ \\
\hline Total & $3120 / 67181$ & 46.44 & & \\
\hline \multicolumn{5}{|c|}{ Panel B: Persistent knee pain } \\
\hline \multicolumn{5}{|c|}{ Depressive symptoms } \\
\hline Without & $314 / 36125$ & 8.69 & 1 [Reference] & 1 [Reference] \\
\hline With & $427 / 14977$ & 28.51 & $3.33(2.88-3.85)$ & $2.16(1.85-2.52)$ \\
\hline Total & $741 / 51102$ & 14.50 & & \\
\hline
\end{tabular}

Notes: Depressive symptoms were defined as a score of 10 or greater on the 10-item Center for Epidemiologic Studies Depression Scale 

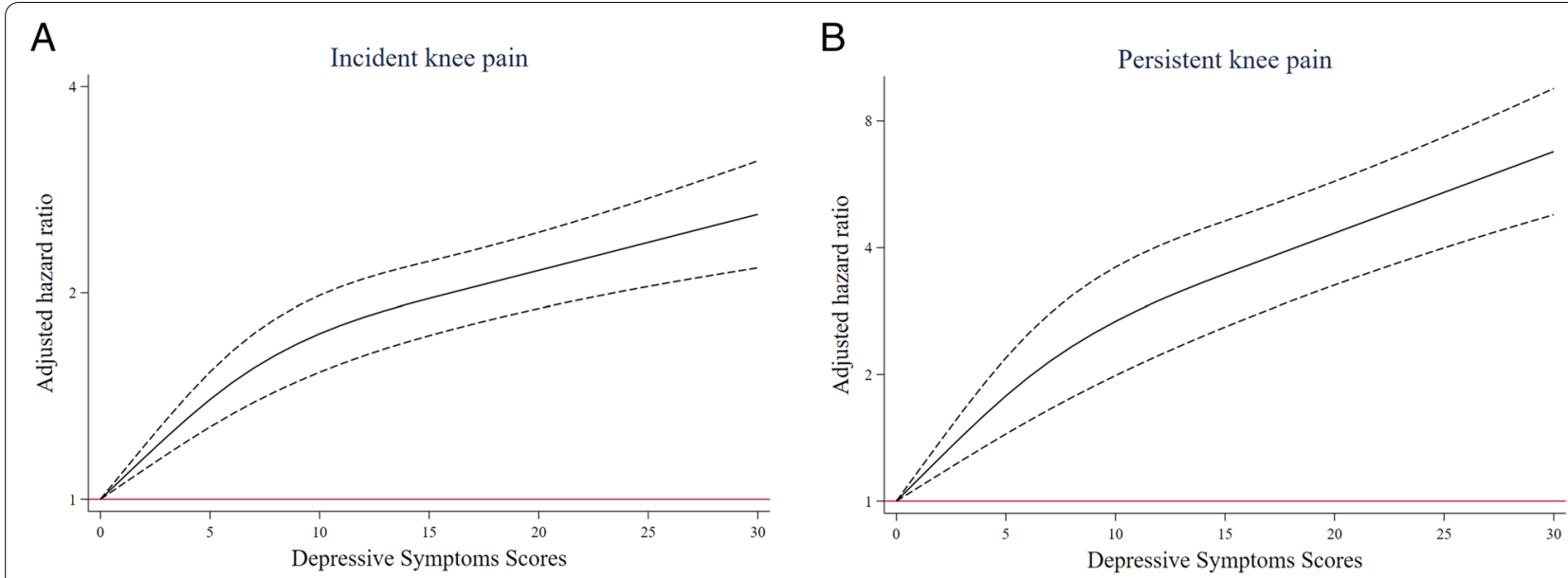

Fig. 2 Adjusted hazard ratios (HRs) of knee pain according to depressive symptoms scores. Notes: Graphs show HRs for incident knee pain (Panel A) and persistent knee pain (Panel B). HRs were adjusted for demographic characteristics, socioeconomic status (SES), health status, and health-related behavior. Data were fitted by a 3-knots restricted cubic spline Cox proportional hazards regression model. The depressive symptoms score ranges from 0 to 30, with the highest score representing the highest risk of depressive symptoms. Solid lines indicate HRs, and dashed lines indicate 95\% $\mathrm{Cls}$

Table 3 Moderating effect of sex on the relationship between depressive symptoms status and the risk of knee pain

\begin{tabular}{ll}
\hline Outcome & Hazard ratio $\mathbf{9 5 \%} \mathbf{C l})$ \\
\hline Panel A: Incident knee pain & \\
Depressive symptoms (Ref = without) & $1.35(1.23-1.48)$ \\
Sex (Ref = female) & $0.66(0.58-0.74)$ \\
Interaction (Depressive symptoms $\times$ Sex) & $1.22(1.05-1.42)$ \\
Panel B: Persistent knee pain & \\
Depressive symptoms (Ref = without) & $1.88(1.57-2.26)$ \\
Sex (Ref = female) & $0.37(0.28-0.49)$ \\
Interaction (Depressive symptoms $\times$ Sex) & $1.57(1.14-2.17)$
\end{tabular}

Notes: Depressive symptoms were defined as a score of 10 or greater on the 10-item Center for Epidemiologic Studies Depression Scale

\section{Depressive symptoms increased the risk of knee pain}

Depressive symptoms were associated with an approximately 1.45-to-2.16-fold higher risk of knee pain after adjustment for confounding factors. This result was comparable with previous findings that depressive symptoms were correlated with an increased risk of knee pain. Wright et al. [16] reported a statistically significant simple correlation coefficient between depressive symptoms and knee pain among 275 knee OA recruited from the urban community. Nevertheless, the sample inclusion limited the generalizability of the results to the general population due to the exclusion of participants with moderate to high levels of physical activity at baseline [16]. Peat and Thomas [18] performed a nested case-control study, which included
57 cases and 228 controls aged over 50 years. They reported that an increase in depressive symptoms was associated with substantial deterioration of knee pain. Riddle et al. [22] found depressive symptoms predictive for worsening self-reported knee pain with a small magnitude effect, indicating depression was required to persist for a considerable year to produce an appreciable impact on future knee pain.

Although multiple studies have investigated the relationship between depressive symptoms and knee pain, the degree to which depressive symptoms are associated with the risk of future knee pain among the most vulnerable middle-aged and elderly general population remains unknown. Most of the current studies were cross-sectional studies [13-17, 20, 21], based on which the temporal direction of such relationships is difficult to discern; some previous studies examined osteoarthritis (OA) patients recruited in the clinics or hospitals $[14,15]$. Therefore, findings from these studies may not be generalizable to the general population. Knee pain is usually under-reported, under-diagnosed, and under-treated, particularly among the communitydwelling middle-aged and older adults [36]. Papandony et al. systematically reviewed patients' perceived health service needs for OA care. They found that increases in frequency and intensity of symptoms and disturbance to their daily life activities may motivate their healthcare-seeking behaviors [37]. It may lead to differences in knee pain intensity, frequency, or interference among participants recruited from medical institutions and community settings. Previous studies have 
also suggested that the association of depressive symptoms and pain would differ by pain characteristics [38]. Therefore, this association might vary among different research populations. The link found in studies focusing on OA patients recruited in the clinics or hospitals could not apply to the general population. The research on the general population may be of more relevance to public health and preventive care. It is particularly significant in light of the prevalence and impact of comorbid depression and knee pain. Specifying this association among the general population would help identify the population at risk and promote primary prevention strategies and secondary care management that may prevent incident knee pain from progressing into chronicity.

The relationship between depressive symptoms and risk of knee pain persisted when the subjects were stratified by the phenotype of knee pain. Specifically, the correlation between depressive symptoms and persistent knee pain (HR: 2.16) was much stronger than that of depressive symptoms and incident knee pain (HR: 1.45). It indicated that the association of depressive symptoms with future knee pain was more evident for chronicity than non-chronicity.

These findings were supported by previous functional studies, which revealed that the emotional processing within the insula in depressed patients was shifted topologically toward the insula areas, which were generally involved in pain processing $[39,40]$. Overlapping between emotional and pain-processing circuitry supported the association between depressive symptoms and pain experience [40]. The changes could last long and thus produce a persistent effect on pain symptoms [41].

Central sensitization could be another explanation for this association [42]. Central sensitization could be manifested clinically as hyperalgesia (exaggerated and prolonged in response to noxious stimuli), secondary allodynia (painful sensation to a normally non-painful stimulus), expansion of the receptive field (pain that extends beyond the area of peripheral nerve supply), and unusually prolonged pain after the stimulus has been removed (usually burning, throbbing, tingling, or numbness) [43]. Therefore, chronic or persistent pain was considered as being maintained in part by central sensitization [44]. However, central sensitization could be driven by neuroinflammation, one of the common pathophysiology for depression and pain [44-47]. Neuroinflammation could be a joint driving force for chronic pain and neuropsychiatric diseases such as depression [44]. This study revealed a significant correlation between depressive symptoms and persistent knee pain. Future researchers would further explore other aspects of central sensitization, such as the relationship between depression and multi-site or widespread pain.

\section{Sex moderated the association of depressive symptoms and the risk of knee pain}

Analysis of moderating variables revealed variations in the magnitude of the depression-knee pain relationship, not the direction of the depression difference. That is, all HRs for the risk of knee pain were more significant than 1.0 among different sexes. These findings indicated that depressive symptoms contributed to future knee pain for both sexes, emphasizing the consistency that depressed respondents had an elevated risk for knee pain than those non-depressed. However, compared with females, males had an enhanced correlation between depression and knee pain (1.22-1.57 times). These results were consistent for different knee pain phenotypes, with the most substantial moderation effect observed for the persistent knee pain (1.57 times).

Evidence suggested that sex differences in the depressive symptoms-pain association may be caused by multiple biological-social-psychological mechanisms, including sex hormones and their interaction with the immune system, gender roles, pain coping, and catastrophizing $[48,49]$. The sex difference in depressive symptoms may also be caused by biological factors, psychological factors, and multiple factors at micro and macro levels [50].

Nevertheless, sex was often controlled as a confounder in the analytic models in previous studies [51]. To date, research on sex differences in the relationship between depressive symptoms and future knee pain was relatively lacking. Perruccio et al. [51] examined the influence of depressive symptoms on post-total knee arthroplasty (TKA) pain among TKA patients. They found that sex moderated the effect of depressive symptoms on postsurgery knee pain, with a more significant effect being observed for males than for females [51]. Our findings were comparable with Perruccio et al. However, our study had a more generalized population, a larger sample size, and a longer follow-up.

The differential correlation strength of depressive symptoms with knee pain between varied sexes, which is more significant for males, did not suggest that depressive symptoms were inconsequential in females. Accumulated evidence indicated that females were at substantially greater risk for depressive symptoms and knee pain [52, 53]. Males may be less likely to suffer from depressive symptoms and painful knees than females; however, this does not mean that depressed males would be less impaired by this correlation. Conservatively speaking, our findings could be interpreted as equal attention should be paid to the males, who were 
in a disadvantaged position in the relationship between depressive symptoms and risk of knee pain. It is very important to avoid overlooking depressive symptoms among males and overemphasizing depressive symptoms among females at the clinical and primary care settings to eliminate or minimize the harm of sex stereotypes, in other words, to refrain from over-diagnosis and overtreatment of females, and under-diagnosis and underreporting of males [53]. Furthermore, the correlation of depressive symptoms with subsequent knee pain cannot be assumed to be the same for different sexes, which may mask or weaken the underlying association for males [51].

\section{Strengths and potential limitations}

This study used a longitudinal research design, adopted a large sample size from community-based rather than general clinics or hospital-based populations, included a significant number of participants who reported symptoms but may not have sought medical help, thereby increasing the generalizability of our findings. However, this study was not without limitation. First, there were $26.56 \%$ of participants ineligible due to missing data on interest variables. Second, measurement errors may perhaps not be entirely excluded and would be another source of bias. Although CESD-10 used to evaluate depression has been validated and shown fair reliability among Chinese community-dwelling adults [29], misclassification of depressive symptoms would also exist due to the unavailability of a clinical diagnostic tool for depression in CHARLS. Knee pain was measured via single self-report through binary options, i.e., yes or no. However, it may be prone to underestimate pain particularly for the older adults who would consider pain to be part of normal age-related physical declines [54]. Third, depressive symptoms would not be fixed. Instead, they could be time-variant, making assessing causality more challenging [55]. The potential reciprocal relationship between depressive symptoms and knee pain should also be noted $[19,56]$. Fourth, our findings were likely to suffer from omitted variable bias that would be caused by unmeasured confounders responsible for the correlation. Therefore, though the longitudinal design was applied, the observational nature of this study did not warrant causal inferences. Our findings should be interpreted in light of, but not restricted to, the above limitations.

\section{Theoretical and practical implications}

Despite these limitations, the findings as a whole have provided valuable information that may have implications for future practice and research. First, this study strengthened the evidence for the correlation of depressive symptoms with knee pain, particularly the chronicity of knee pain complaints among the general middle-aged and elderly population. It would be helpful to develop and provide psychological preventive and treatment programs. As the population ages, the direct and indirect costs associated with knee pain may also shift upwards, highlighting the importance of identifying those at higher risk, thereby promoting primary prevention strategies and secondary care management that may prevent incident knee pain from progressing into chronicity.

Second, the findings suggest the association of depressive symptoms with knee pain risk would be different by sexes. Therefore, it would be necessary to notice the sex-moderated association of depressive symptoms with knee pain and minimize the harm of sex stereotypes. Though males were less likely to suffer from knee pain or depressive symptoms than females, depressed males had a higher risk of knee pain than depressed females. Therefore, it is crucial to give males an adequate psychological evaluation. It is also imperative to advocate a more personalized approach to shared public health and prevention decisions in rural and urban areas, considering local cultures and resources to achieve better healthcare results from a group perspective.

Third, psychological assessments and interventions should be more accessible in the primary care settings closest to the people at risk. More efforts are needed in public education of psychological services, mental health, well-being, and local resources for promoting healthy living.

Finally, for future research, an essential next step is to design and evaluate the potential beneficial effects of preventive strategies for those at risk of knee pain and continue to improve the administration of prevention efforts. Further research is needed to compare with our findings and explain why these sex differences exist. In addition, it should be taken into account that the complexity of accurate pain assessment strategies caused by aging-related physiological changes when using knee pain assessment tools in future studies [54].

\section{Conclusion}

This study found a positive correlation between depressive symptoms and the risk of knee pain among middleaged and elderly Chinese adults. This correlation was moderated by sex, with a stronger association among males than females.

\section{Abbreviations}

MSK: Musculoskeletal; DALYs: Disability-adjusted life-years; CHARLS: China Health and Retirement Longitudinal Study; CESD: Center for Epidemiological Studies-Depression Scale; SES: Socioeconomic status; BMI: Body mass index; SD: Standard deviation; Cl: Confidence interval; HR: Hazard ratio; MAR: Missing at random; OA: Osteoarthritis; TKA: Total knee arthroplasty. 


\section{Supplementary Information}

The online version contains supplementary material available at https://doi. org/10.1186/s12891-021-04511-2.

Additional file 1: Appendix Table 1. Hazard ratio (HR) of knee pain according to depressive symptoms status after multiple imputation

Additional file 2: Appendix Table 2. Moderated effect of sex on the association of depressive symptoms status and knee pain

Additional file 3. Association between depressive symptoms and risk of incident knee pain

Additional file 4. Association between depressive symptoms and risk of persistent knee pain

\section{Acknowledgements}

We sincerely thank the China Center for Economic Research, National School of Development at Peking University, for sharing the China Health and Retirement Longitudinal Study (CHARLS) data generously. We are grateful to all the interviewees who participated in the survey. We appreciate Dr. Yuehong Chen Foley for proofreading our manuscript.

\section{Authors' contributions}

All authors contributed to the conception of the study, drafting, revising the manuscript, and gave final approval of the version to be published. Haiyan $\mathrm{Hu}$ and Wenjun Liu designed the study, analyzed the data, and drafted the initial manuscript. Haiyan Hu had full access to all the data in the research and took responsibility for the data integrity and the accuracy of the data analyses. Yang Liu took part in the interpretation of the results. Jay Pan and Xiaozuo Zheng critically reviewed the manuscript for important intellectual content.

\section{Funding}

This work was supported by the National Natural Science Foundation of China (Grant No. 71874116 and 72074163), Chengdu Federation of Social Science Association (Grant No. ZZ05), Sichuan University (Grant No. 2018hhf-27 and SKSYL201811), China Medical Board (Grant No. 17-276) and Medical Science of Hebei Province (Grant No. 20190645).

\section{Availability of data and materials}

The datasets used and/or analyzed during the current study are available from the corresponding author on reasonable request.

\section{Declarations}

\section{Ethics approval and consent to participate}

Written informed consent was obtained from all the participants, and ethical approval for collecting data on human subjects was received from the Biomedical Ethics Review Committee of Peking University (IRB00001052-11015) [28]. Ethics approval for the use of CHARLS data was obtained from the University of Newcastle Human Research Ethics Committee (H-2015-0290).

\section{Consent for publication}

Not applicable.

\section{Competing interests}

The authors report no conflicts of interest in this work.

\section{Author details}

${ }^{1}$ HEOA Group, West China School of Public Health and West China Fourth Hospital, Sichuan University, No. 16, Section 3, Ren Min Nan Road, Chengdu 610041, China. ${ }^{2}$ Department of Spine Surgery, The First College of Clinical Medical Science, China Three Gorges University, Yichang 443003, Hubei, China. ${ }^{3}$ Department of Spine Surgery, Yichang Central People's Hospital, Yichang 443003, Hubei, China. ${ }^{4}$ Institute for Healthy Cities and West China Research Center for Rural Health Development, Sichuan University, Chengdu 610041, Sichuan, China. ${ }^{5}$ Department of Orthopaedic Surgery, Third Hospital of Hebei Medical University, No.139 Ziqiang Road, Shijiazhuang 050051, Hebei, China.
Received: 1 February 2021 Accepted: 7 July 2021

Published online: 26 July 2021

\section{References}

1. Bunt CW, Jonas CE, Chang JG. Knee pain in adults and adolescents: the initial evaluation. Am Fam Phys. 2018;98:576-85.

2. Dawson J, Linsell L, Zondervan K, Rose P, Randall T, Carr A, Fitzpatrick R. Epidemiology of hip and knee pain and its impact on overall health status in older adults. Rheumatology (Oxford). 2004;43:497-504.

3. Yoshimura N, Akune T, Fujiwara S, Shimizu Y, Yoshida H, Omori G, Sudo A, Nishiwaki Y, Yoshida M, Shimokata $H$, et al. Prevalence of knee pain, lumbar pain and its coexistence in Japanese men and women: The Longitudinal Cohorts of Motor System Organ (LOCOMO) study. J Bone Miner Metab. 2014;32:524-32.

4. Ballantyne JC, Fishman SM, Rathmell JP. Bonica's management of pain. 5th ed. Philadelphia: Wolters Kluwer Health; 2018.

5. Neogi T. The epidemiology and impact of pain in osteoarthritis. Osteoarthritis Cartilage. 2013:21:1145-53.

6. Creamer P, Hochberg MC. The relationship between psychosocial variables and pain reporting in osteoarthritis of the knee. Arthritis Care Res. 1998;11:60-5.

7. Sarmanova A, Fernandes GS, Richardson H, Valdes AM, Walsh DA, Zhang W, Doherty M. Contribution of central and peripheral risk factors to prevalence, incidence and progression of knee pain: a community-based cohort study. Osteoarthritis Cartilage. 2018;26:1461-73.

8. Phyomaung PP, Dubowitz J, Cicuttini FM, Fernando S, Wluka AE, Raaijmaakers P, Wang Y, Urquhart DM. Are depression, anxiety and poor mental health risk factors for knee pain? A systematic review. BMC Musculoskelet Disord. 2014;15:10

9. GBD 2019 Diseases and Injuries Collaborators. Global burden of 369 diseases and injuries in 204 countries and territories, 1990-2019: a systematic analysis for the Global Burden of Disease Study 2019. Lancet. 2020:396:1204-22.

10. Dowlati Y, Herrmann N, Swardfager W, Liu H, Sham L, Reim EK, Lanctot $\mathrm{KL}$. A meta-analysis of cytokines in major depression. Biol Psychiatry. 2010;67:446-57.

11. Dieppe PA, Lohmander LS. Pathogenesis and management of pain in osteoarthritis. Lancet. 2005:365:965-73.

12. Suzuki R, Rygh $L$, Dickenson AH. Bad news from the brain: descending 5-HT pathways that control spinal pain processing. Trends Pharmacol Sci. 2004;25:613-7

13. Creamer P, Lethbridge-Cejku M, Costa P, Tobin JD, Herbst JH, Hochberg $M C$. The relationship of anxiety and depression with self-reported knee pain in the community: data from the Baltimore Longitudinal Study of Aging. Arthritis Care Res. 1999;12:3-7.

14. Creamer $P$, Lethbridge-Cejku M, Hochberg MC. Determinants of pain severity in knee osteoarthritis: effect of demographic and psychosocial variables using 3 pain measures. J Rheumatol. 1999:26:1785-92.

15. van Baar ME, Dekker J, Lemmens JA, Oostendorp RA, Bijlsma JW. Pain and disability in patients with osteoarthritis of hip or knee: the relationship with articular, kinesiological, and psychological characteristics. J Rheumatol. 1998;25:125-33.

16. Wright LJ, Zautra AJ, Going S. Adaptation to early knee osteoarthritis: the role of risk, resilience, and disease severity on pain and physical functioning. Ann Behav Med. 2008;36:70-80.

17. Han HS, Lee JY, Kang SB, Chang CB. The relationship between the presence of depressive symptoms and the severity of self-reported knee pain in the middle aged and elderly. Knee Surg Sports Traumatol Arthrosc. 2016:24:1634-42.

18. Peat $G$, Thomas E. When knee pain becomes severe: a nested case-control analysis in community-dwelling older adults. J Pain. 2009;10:798-808.

19. Kroenke K, Wu J, Bair MJ, Krebs EE, Damush TM, Tu W. Reciprocal relationship between pain and depression: a 12-month longitudinal analysis in primary care. J Pain. 2011;12:964-73.

20. Han SB, Lee SH, Ha IH, Kim EJ. Association between severity of depressive symptoms and chronic knee pain in Korean adults aged over 50 years: a cross-sectional study using nationally representative data. BMJ Open. 2019:9:e32451. 
21. Kim KW, Han JW, Cho HJ, Chang CB, Park JH, Lee JJ, Lee SB, Seong SC, Kim TK. Association between comorbid depression and osteoarthritis symptom severity in patients with knee osteoarthritis. J Bone Joint Surg Am. 2011;93:556-63.

22. Riddle DL, Kong X, Fitzgerald GK. Psychological health impact on 2-year changes in pain and function in persons with knee pain: data from the Osteoarthritis Initiative. Osteoarthritis Cartilage. 2011;19:1095-101.

23. Van de Velde S, Bracke P, Levecque K, Meuleman B. Gender differences in depression in 25 European countries after eliminating measurement bias in the CES-D 8. Soc Sci Res. 2010;39:396-404.

24. Luppa M, Sikorski C, Luck T, Ehreke L, Konnopka A, Wiese B, Weyerer S, Konig HH, Riedel-Heller SG. Age- and gender-specific prevalence of depression in latest-life-systematic review and meta-analysis. J Affect Disord. 2012;136:212-21.

25. Greenspan JD, Craft RM, LeResche L, Arendt-Nielsen L, Berkley KJ, Fillingim RB, Gold MS, Holdcroft A, Lautenbacher S, Mayer EA, et al. Studying sex and gender differences in pain and analgesia: a consensus report. Pain. 2007:132(Suppl 1):S26-45.

26. Manson JE. Pain: sex differences and implications for treatment. Metabolism. 2010;59(Suppl 1):S16-20.

27. Zhao Y, Strauss J, Chen X, Wang Y, Gong J, Meng Q, Wang G, Wang H. China health and retirement longitudinal study wave 4 user's guide.: National School of Development, Peking University; 2020

28. Zhao Y, Hu Y, Smith JP, Strauss J, Yang G. Cohort profile: the China Health and Retirement Longitudinal Study (CHARLS). Int J Epidemiol. 2014:43:61-8.

29. Xue Q, Pan A, Gong J, Wen Y, Peng X, Pan J, Pan X. Association between arthritis and depression risk: a prospective study and meta-analysis. J Affect Disorders. 2020;273:493-9.

30. Zhao F, Pan A, Yang X, Meng R, Ye Y, Wang Y, Xie Y, Pan X, Yang C. Bidirectional association between depressive symptoms and type 2 diabetes mellitus: The China Health and Retirement Longitudinal Study. J Diabetes Complicat. 2019;33:107387.

31. Andresen EM, Malmgren JA, Carter WB, Patrick DL. Screening for depression in well older adults: evaluation of a short form of the CES-D (Center for Epidemiologic Studies Depression Scale). Am J Prev Med. 1994;10:77-84.

32. Chen $\mathrm{H}$, Mui AC. Factorial validity of the center for epidemiologic studies depression scale short form in older population in China. Int Psychogeriatr. 2014;26:49-57.

33. Hu H, Liu W, Zhang S, Pan J, Zheng X. Depression mediates the relationship between smoking and pain: evidence from a nationally representative study in a low- and middle-income country. Addict Behav. 2021;119:106937.

34. Zhou BF, Cooperative Meta-Analysis Group of the Working Group on Obesity in China. Predictive values of body mass index and waist circumference for risk factors of certain related diseases in Chinese adults--study on optimal cut-off points of body mass index and waist circumference in Chinese adults. Biomed Environ Sci. 2002;15:83-96.

35. Cleves M, Gould W, Gutierrez R, Marchenko Y. An introduction to survival analysis using Stata. 3rd ed. College Station, Texas: Stata Press; 2010.

36. Barkin RL, Barkin SJ, Barkin DS. Pharmacotherapeutic management of pain with a focus directed at the geriatric patient. Rheum Dis Clin North Am. 2007;33:1-31.

37. Papandony MC, Chou L, Seneviwickrama M, Cicuttini FM, Lasserre K, Teichtahl AJ, Wang Y, Briggs AM, Wluka AE. Patients' perceived health service needs for osteoarthritis (OA) care: a scoping systematic review. Osteoarthritis Cartilage. 2017;25:1010-25.

38. de Heer EW, Gerrits MM, Beekman AT, Dekker J, van Marwijk HW, de Waal MW, Spinhoven P, Penninx BW, van der Feltz-Cornelis CM. The association of depression and anxiety with pain: a study from NESDA. Plos One. 2014:9:e106907.
39. Borsook D, Sava S, Becerra L. The pain imaging revolution: advancing pain into the 21st century. Neuroscientist. 2010;16:171-85.

40. Mutschler I, Ball T, Wankerl J, Strigo IA. Pain and emotion in the insular cortex: evidence for functional reorganization in major depression. Neurosci Lett. 2012;520:204-9.

41. Gerrits MMJG, van Marwijk HWJ, van Oppen P, van der Horst H, Penninx BWJH. Longitudinal association between pain, and depression and anxiety over four years. J Psychosom Res. 2015;78:64-70.

42. den Boer C, Dries L, Terluin B, van der Wouden JC, Blankenstein AH, van Wilgen CP, Lucassen P, van der Horst HE. Central sensitization in chronic pain and medically unexplained symptom research: a systematic review of definitions, operationalizations and measurement instruments. J Psychosom Res. 2019;117:32-40.

43. Mayer TG, Neblett R, Cohen H, Howard KJ, Choi YH, Williams MJ, Perez Y, Gatchel RJ. The development and psychometric validation of the central sensitization inventory. Pain Pract. 2012;12:276-85.

44. Ji RR, Nackley A, Huh Y, Terrando N, Maixner W. Neuroinflammation and central sensitization in chronic and widespread pain. Anesthesiology. 2018;129:343-66

45. Maletic V, Raison CL. Neurobiology of depression, fibromyalgia and neuropathic pain. Front Biosci (Landmark Ed). 2009;14:5291-338.

46. Kiecolt-Glaser JK, Derry HM, Fagundes CP. Inflammation: depression fans the flames and feasts on the heat. Am J Psychiat. 2015;172:1075-91.

47. Slavich GM, Irwin MR. From stress to inflammation and major depressive disorder: a social signal transduction theory of depression. Psychol Bull. 2014;140:774-815.

48. Oon-Arom A, Likhitsathian S, Maneeton B, Sulaiman AH, Shih-Yen EC, Udomratn $\mathrm{P}$, Chen $\mathrm{CH}$, Srisurapanont M. Subjective depressive symptoms associated with pain in patients with major depressive disorder: findings from the study on the aspect of Asian depression. Perspect Psychiatr Care. 2020;56:188-93.

49. Sorge RE, Totsch SK. Sex differences in pain. J Neurosci Res. 2017:95:1271-81.

50. Kuehner $\mathrm{C}$. Why is depression more common among women than among men? Lancet Psychiatry. 2017;4:146-58.

51. Perruccio AV, Fitzpatrick J, Power JD, Gandhi R, Rampersaud YR, Mahomed NN, Davey JR, Syed K, Veillette C, Badley EM. Sex-modified effects of depression, low back pain, and comorbidities on pain after total knee arthroplasty for osteoarthritis. Arthritis Care Res (Hoboken). 2020;72:1074-80

52. Fillingim RB, King CD, Ribeiro-Dasilva MC, Rahim-Williams B, Riley JL. Sex, gender, and pain: a review of recent clinical and experimental findings. J Pain. 2009;10:447-85.

53. Salk RH, Hyde JS, Abramson LY. Gender differences in depression in representative national samples: meta-analyses of diagnoses and symptoms. Psychol Bull. 2017;143:783-822.

54. Kang Y, Demiris G. Self-report pain assessment tools for cognitively intact older adults: integrative review. Int J Older People Nurs. 2018;13:e12170.

55. VanderWeele TJ, Jackson JW, Li S. Causal inference and longitudinal data: a case study of religion and mental health. Soc Psychiatry Psychiatr Epidemiol. 2016;51:1457-66.

56. Angst F, Benz T, Lehmann S, Wagner S, Simmen BR, Sandor PS, Gengenbacher M, Angst J. Extended overview of the longitudinal pain-depression association: a comparison of six cohorts treated for specific chronic pain conditions. J Affect Disord. 2020;273:508-16.

\section{Publisher's Note}

Springer Nature remains neutral with regard to jurisdictional claims in published maps and institutional affiliations. 